\title{
Non balla ma uccide
}

\section{Not moving, but it kills}

Giovanni Lodi

Dipartimento di Scienze Biomediche, Chirurgiche e Odontoiatriche

Università degli Studi di Milano

via Beldiletto 1/3 Milano 20142

Italia

giovanni.lodi@unimi.it

tel. + 390250319021

fax. +390250319041

Appare evidente, sulla base dei dati raccolti da una autorevole agenzia statunitense, che un omicida seriale abbia agito indisturbato per tre lustri, spezzando venticinque vite innocenti.

Come nella trama di un noir neanche troppo originale, il colpevole è assolutamente insospettabile. Presenza familiare e rassicurante, ha avvelenato le vittime, quasi tutte anziane e in precarie condizioni di salute, lentamente, in maniera subdola, senza che se accorgessero. Fino a quando è stato troppo tardi.

Dubito che questi pochi elementi siano stati sufficiente a farvi indovinare il colpevole. E anche aggiungere che l'agenzia in questione è la Food and Drug Administration, ovvero l'ente statunitense che regolamenta prodotti alimentari e farmaci, potrebbe non essere abbastanza. L'elemento chiave per risolvere il mistero è un altro e si trova nella bocca delle vittime: tutti portatori di protesi totale. Sì perché l'assassino è la pasta adesiva per la dentiera.

Il meccanismo alla base di queste morti, e verosimilmente di tanti altri casi meno gravi, è un'intossicazione da zinco, capace di causare malattie neurologiche e del tessuto emopoietico.

Nessun allarme e nessun allarmismo. I produttori sono corsi ai ripari e lo zinco è scomparso dalla formulazione di molte paste adesive (ma non di tutte) e probabilmente solo un uso veramente sconsiderato è in grado di fare danni.

Il punto è che gli eventi avversi rimangono un oggetto misterioso della medicina. Di sicuro sappiamo che non esistono trattamenti privi di rischi. E storie come queste ci insegnano che questo vale anche per quelli apparentemente più innocui. Ma le certezze finiscono qua. La ricerca in questo campo è difficile da condurre, interessa a pochi, e nessuno è disposto a finanziarla.

Mi chiedo se questo sia accettabile, in particolare per trattamenti che quotidianamente vengono proposti a migliaia di pazienti. Quanto sono frequenti i danni causati dagli impianti? esiste l'allergia alle leghe dentarie? quali sono i rischi dei biomateriali? E se per queste domande non abbiamo risposte, su cosa è informato il consenso che otteniamo dai nostri pazienti?

\section{Buona lettura}

